Full length article

\title{
Physicochemical study of biomolecular interactions between lysosomotropic surfactants and bovine serum albumin
}

\author{
Tomasz Janek $^{\mathrm{a}, *}$, Żaneta Czyżnikowska ${ }^{\mathrm{a}}$, Jacek Łuczyński ${ }^{\mathrm{b}}$, Eduardo J. Gudiña ${ }^{\mathrm{c}}$, \\ Lígia R. Rodrigues ${ }^{c}$, Joanna Gałęzowska ${ }^{a}$ \\ a Department of Inorganic Chemistry, Faculty of Pharmacy, Wroclaw Medical University, Borowska 211a, 50-556, Wroclaw, Poland \\ ${ }^{b}$ Department of Organic and Pharmaceutical Technology, Faculty of Chemistry, Wroclaw University of Science and Technology, Wybrzeze Wyspianskiego \\ 27, 50-370, Wroclaw, Poland \\ ${ }^{\mathrm{c}}$ CEB - Centre of Biological Engineering, University of Minho, 4710-057, Braga, Portugal
}

\section{A R T I C L E I N F O}

\section{Article history:}

Received 9 March 2017

Received in revised form 5 August 2017

Accepted 25 August 2017

\section{Keywords:}

Lysosomotropic substances

Surface tension

Density functional theory

Fluorescence quenching

Circular dichroism

Isothermal titration calorimetry

\begin{abstract}
A B S T R A C T
The interactions between two cationic lysosomotropic surfactants (2-dodecanoyloxyethyl)trimethylammonium bromide (DMM-11) and (2dodecanoyloxypropyl)trimethylammonium bromide (DMPM-11) with bovine serum albumin (BSA) in Hepes buffer $(\mathrm{pH}=7.4)$ were systematically studied by surface tension, fluorescence and circular dichroism (CD) spectroscopy and isothermal titration calorimetry (ITC). Furthermore, the size of the micellar aggregates and the polydispersity indexes of both cationic surfactants were studied by dynamic light scattering technique (DLS). The hydrodynamic radii, micellar volumes and aggregation numbers were calculated using a method based on density functional theory (DFT). The results showed that, in both cases, the surface tension was modified upon addition of BSA, and the critical micelle concentration (CMC) values of DMM-11 and DMPM-11 were higher in the presence of BSA. The fluorescence intensity of BSA decreased significantly as the concentration of both cationic surfactants increased and this effect was attributed to the formation of surfactant-BSA complexes. Synchronous fluorescence spectrometry showed the binding-induced conformational changes in BSA. Finally, CD and DLS results revealed the occurrence of changes in the secondary structure of the protein in the presence of both surfactants. In conclusion, understanding the interactions between lysosomotropic surfactants and BSA is required to explore their potential applications in medicine.
\end{abstract}

(c) 2017 Elsevier B.V. All rights reserved.

\section{Introduction}

Long-chain quaternary ammonium surfactants (QACs) exert antibacterial activity against both Gram-positive and Gramnegative bacteria, as well as against some viruses and pathogenic species of fungi and protozoa [1]. However, these cationic surfactants are known to cause various side effects, including toxic effects against mammalian cells. Consequently, long-chain QACs are not recommended for systemic application [2], but are considered acceptable for topical application (e.g. mouthwash products and oral antiseptics). Their adverse effects can be avoided by the use of soft analogues of long-chain QACs, which are synthesized according to the soft drug approach and their physicochemi-

\footnotetext{
* Corresponding author at: Department of Inorganic Chemistry, Faculty of Pharmacy, Wroclaw Medical University, Borowska 211a, 50-556, Wroclaw, Poland.

E-mail address: tomasz.janek@umed.wroc.pl (T. Janek).
}

cal properties, such as their surface activity and lipophilicity [3]. Several soft cationic QACs have been synthesized, such as alkyl $N, N$-dimethylalaninates methobromides (DMALM- $n$ ), alkyl $N, N$-dimethylglycinates methobromides (DMGM- $n$ ), fatty acids $N, N$-dimethylaminoethylesters methobromides (DMM- $n$ ) and fatty acids $\mathrm{N}, \mathrm{N}$-dimethylaminopropylesters methobromides (DMPM-n) [4], which showed satisfactory antibacterial and antifungal properties, as well as good chemical stability [5-7]. Due to their properties, soft cationic QACs might be used in lysosome-targeting anti-cancer drugs and drug delivery systems.

A number of soft cationic surfactants exhibit pronounced effects in biological systems [8]; therefore, the study of protein-surfactant interactions is a relevant and timely topic. The specific and nonspecific interactions between surfactants and proteins have been a subject of extensive studies over the past decades, due to their significance in drug delivery, cosmetic preparation, biosciences and foods [9-11]. Studies on the interactions of cationic, anionic, zwitterionic and nonionic surfactants with globular proteins such 
as human serum albumin (HSA) [12] and bovine serum albumin (BSA) $[13,14]$ can contribute towards a better understanding of the action of surfactants as solubilizing agents of membranes containing proteins and lipids [15]. BSA is commonly used as an expedient protein and is widely implicated as a general model to study the interactions of surfactants [14] and drugs [16] with globular proteins, not only due to its important roles in biological processes, but also because its structure is well established. BSA can bind many different types of amphiphilic biological molecules, which are believed to play an important role in determining its physiological functions. While a large number of studies are devoted to the interactions between proteins and traditional long-chain cationic surfactants, studies regarding soft QACs-protein systems are scarce. As a new class of amphiphilic molecules, soft cationic surfactants exhibit good biocompatibility and degradability, as well as less toxicity than traditional cationic surfactants. Liu and co-workers [17] studied the interactions of HSA with sodium dodecyl sulfate (SDS) and cetyl trimethylammonium bromide (CTAB) using fluorescence spectroscopy. The authors suggested that hydrophobic and electrostatic forces play an important role in protein-surfactant interactions. Dodecyl trimethylammonium bromide (DTAB), tetradecyltrimethylammonium bromide (TTAB) and $\mathrm{CTAB}$ have been reported to act as chemical chaperones, aiding in the refolding of proteins via hydrophobic interactions [18].

Given the growing interest of new biomedical applications for lysosomotropic QACs, the aim of this work is to present a detailed molecular study of the interactions between two cationic lysosomotropic surfactants (DMM-11 and DMPM-11) and BSA, a model globular protein, in order to characterize the basic features of these compounds and the potential application of lysosomotropic surfactants in medicine and drug delivery. It is worth mentioning that DMM-11 and DMPM-11 belong to the so-called soft lysosomotropic compounds, which are easily degraded by the cells to nontoxic metabolites.

To the best of our knowledge, this is the first report on the molecular interactions between DMM-11/DMPM-11 and BSA, as evaluated by surface tension and fluorescence spectroscopy measurements. The effect of the head groups of both cationic surfactants on the secondary structure of BSA was examined by circular dichroism (CD) spectroscopy. Dynamic light scattering (DLS) was carried out to study the microstructures of the surfactant-BSA complexes at various DMM-11/DMPM-11 concentrations. Isothermal titration calorimetry (ITC) was used to quantify the gradual increase in the number of surfactant molecules bound per BSA molecule, and to obtain the thermodynamic characteristics of the interactions. The formation of the aggregates at the molecular level and different characteristic parameters of the aggregates such as their size, surface charge and aggregation number were examined. Moreover, the experimental data were supported by the results of density functional theory (DFT) calculations.

\section{Material and methods}

\subsection{Chemicals}

Two derivatives of lysosomotropic substances obtained by quaternization of the appropriate tertiary amino compounds with methyl bromide were studied: (2dodecanoyloxyethyl)trimethylammonium bromide (DMM-11) and (2-dodecanoyloxypropyl)trimethylammonium bromide (DMPM-11). The synthesis of these lysosomotropic surfactants was described in detail in previous studies $[4,19]$, and their chemical structures are presented in Fig. S1. The results concerning the elemental analysis and the ${ }^{1} \mathrm{H}-\mathrm{NMR}$ chemical shifts were collected in Table S1. All the reagents and solvents used were of high grade and were used as received. Bovine serum albumin (BSA) was purchased from Sigma-Aldrich Chemical Co. (St. Louis, $\mathrm{MO})$. All the solutions were prepared in $5 \mathrm{mM}$ Hepes buffer $(\mathrm{pH}$ 7.4) (Sigma-Aldrich) supplemented with $150 \mathrm{mM} \mathrm{NaCl}$ (POCh). The buffer was prepared by dissolving the desired amount of Hepes and $\mathrm{NaCl}$ in deionized water $(>18 \Omega)$. All stock solutions of DMM-11, DMPM-11 and BSA were made dissolving them in Hepes buffer $(5 \mathrm{mM})$, filtered through $0.2 \mu \mathrm{m}$ pore size filters and stored at $4{ }^{\circ} \mathrm{C}$. The concentration of BSA was determined spectrophotometrically at $280 \mathrm{~nm}$ using the molar extinction coefficient $\varepsilon=44$ $720 \mathrm{M}^{-1} \mathrm{~cm}^{-1}$. All the experiments were performed at $\sim 25^{\circ} \mathrm{C}$.

\subsection{Surface tension measurements}

The surface tension measurements were performed using a Krüss K20 Tensiometer (Krüss GmbH, Hamburg, Germany) at $25^{\circ} \mathrm{C}$, according to the du Noüy's ring method [20]. Surfactants and BSA were dissolved in Hepes buffer and mixed to obtain several mixtures containing a constant BSA concentration $(1 \mathrm{~g} / \mathrm{L})$ but different DMM-11 and DMPM-11 concentrations. All samples were allowed to stabilize for $30 \mathrm{~min}$, and afterwards the surface tension values were recorded. The surface tension of the control sample ( $5 \mathrm{mM}$ Hepes buffer ( $\mathrm{pH} 7.4$ ) supplemented with $150 \mathrm{mM} \mathrm{NaCl}$ ) was $70.8 \mathrm{mN} / \mathrm{m}$. The average equilibrium surface tension values were obtained by measuring each sample in triplicate. The surface tension data were analyzed using the Origin software provided with the equipment, to obtain the critical micelle concentration (CMC) values, determined as the breakpoint in the plot representing the surface tension versus surfactant concentration.

\subsection{Fluorescence measurements}

The fluorescence quenching measurements were performed on a Cary Eclipse Fluorescence Spectrophotometer. To study the effect of the addition of DMM-11 and DMPM-11 on the intrinsic fluorescence of $B S A$, the fluorescence spectra of solutions containing a fixed concentration $(15 \mu \mathrm{M})$ of BSA and various surfactant concentrations were monitored using a $1 \mathrm{~cm}$ path length quartz cell. Fluorescence emission spectra in the range $300-400 \mathrm{~nm}$ were measured at an excitation wavelength of $280 \mathrm{~nm}$. Both excitation and emission slit widths were fixed at $5 \mathrm{~nm}$.

Synchronous fluorescence spectra were acquired using the same spectrophotometer. The synchronous fluorescence characteristics of surfactant-BSA complexes were measured at $25^{\circ} \mathrm{C}$, keeping the difference between excitation wavelength and emission wavelength fixed $\left(\Delta \lambda=\lambda_{\mathrm{em}}-\lambda_{\mathrm{ex}}\right)$. For $\Delta \lambda=20 \mathrm{~nm}$, the spectral characteristics information of tyrosine (Tyr) residues were evident while for $\Delta \lambda=60 \mathrm{~nm}$, the spectral characteristics of tryptophan (Trp) residues were observed. All the excitation and emission slits were set at 10 and $2.5 \mathrm{~nm}$, respectively.

\subsection{Circular dichroism (CD) spectroscopy}

CD spectra of BSA and surfactant-BSA complexes in Hepes buffer were recorded on a Jasco model J-1500 spectropolarimeter (JASCO, Tokyo, Japan) at $25^{\circ} \mathrm{C}$ under a constant flow of nitrogen gas. A cell with a $0.1 \mathrm{~cm}$ path length was used for spectra recorded between 200 and $260 \mathrm{~nm}$ with sampling points every $2 \mathrm{~nm}$. A spectrum of the buffer solution was taken and subtracted from the spectra of BSA and surfactant-BSA solutions. The spectra represent the average of nine scans. $\mathrm{CD}$ intensities are expressed in $\Delta \varepsilon\left(\mathrm{dm}^{3} \mathrm{M}^{-1} \mathrm{~cm}^{-1}\right)$. The analysis of secondary structural contents was performed using K2D3 web server. K2D3 web server is an online tool used to assess the secondary structural elements in the form of $\alpha$-helix and $\beta$ strand from the far-UV CD spectra ranging from 190 to $240 \mathrm{~nm}$ [21]. 


\subsection{Isothermal titration calorimetry (ITC) measurements}

ITC experiments were carried out using a Nano ITC calorimeter (TA Instruments) with a standard volume of $1.0 \mathrm{ml}$ cell at $25^{\circ} \mathrm{C}$. All samples were degassed before the analysis. The reference cell was filled with water. In a typical experiment, the solution of BSA (0.09-0.22 mM) was placed in the cell, and each time, a freshly prepared solution of DMM-11 or DMPM-11 (1.0-5.1 mM) was taken in a $250 \mu \mathrm{L}$ injection syringe. A total of 50 injections $(4.85 \mu \mathrm{L}$ each) were added after the calorimeter finalized the primary equilibration, with 200-300 s intervals between the injections, leaving $200 \mathrm{~s}$ at the beginning of the experiment without injection. The stirring rate was set at $300 \mathrm{rpm}$. Longer delays between injections did not affect the results obtained. The calorimeter was operated using the Nano ITC Run software and all the data obtained were analyzed with the NanoAnalyze v. 3.1.2 program. The 'Independent model' and 'Multiple Sites' models were used to evaluate the results. Control experiments were performed in each case; the enthalpies of reagents dilution were subtracted from the enthalpies of binding processes. Each ITC data was collected by two independent measurements. Standard deviations around the fit for each titration are shown in Fig. S2.

\subsection{Dynamic light scattering (DLS)}

The mean particle size and polydispersity indexes (PdI) of the cationic surfactant aggregates (with and without BSA) were estimated by the DLS method. The measurements were performed using a Zetasizer Nano-ZS from Malvern Instruments (UK) at $25^{\circ} \mathrm{C}$. The polydispersity index relates with the particle size distribution, which here ranged from 0 for monodispersed to 1.0 for entirely heterodispersed emulsions. Before the measurements, the samples were filtered through a $0.2 \mu \mathrm{m}$ pore size filter directly into the optical cell to remove any impurity. Each sample was analyzed nine times and information on size distribution by intensity, number and volume was recorded.

\subsection{Computational details}

All the calculations were performed using a method based on Density Functional Theory (DFT). Geometry of investigated surfactants was fully optimized using range-separated CAM-B3LYP exchange-correlation functional [22-24]. The $6-31++G(d, p)$ basis set was used on all the atoms. The geometry optimization was followed by evaluation of hessian matrix to confirm that the obtained structures correspond to minima on the potential energy surface. In all optimizations, the polarizable continuum model (PCM) was adopted to implicitly account for solvent effects (water and Hepes buffer) $[25,26]$. The molecular volumes $\left(V_{\text {mon }}{ }^{\mathrm{DFT}}\right)$ of DMM-11 and DMPM-11 monomers were defined as the volume inside a contour of 0.001 electrons/Bohr3 density. All the calculations were carried out using the Gaussian 09 suite of programs [27]. The length of the micellar radii $\left(R_{\mathrm{H}}{ }^{\mathrm{DFT}}\right)$ was taken as the distance between the farthest nitrogen and the carbon of the terminal methyl group. In order to calculate the aggregation numbers $\left(N_{\mathrm{agg}}\right)$, the $R_{\mathrm{H}}{ }^{\mathrm{DFT}}$ and $V_{\text {mon }}{ }^{\text {DFT }}$ were used, assuming the spherical shape of micelles. The biological activity simulations were performed using a combination of the 3D/4D QSAR BiS/MC and CoCon algorithms developed by ChemoSophia company [28-30].

\section{Results and discussion}

\subsection{Surface tension studies}

Surface tension measurement is a simple and effective method to study the interactions between macromolecules and surfactants
[31]. In this study, the surface tension profiles were obtained for both surfactant systems alone and in the presence of BSA, showing the characteristic break points in the surfactant-BSA systems (Fig. 1). In the case of DMM-11 and DMPM-11 without BSA, CMC values can be observed as the saturation of the interface is reached. A minimum surface tension value was found on the $\gamma-c$ curve for both surfactants, which may be caused by the presence of impurities in the surfactant samples. Additionally, the shapes of the plots around the CMC confirm that both surfactants contain impurities. On the other hand, this effect can be caused by partial hydrolysis of esterquat surfactants. Recent studies revealed that cationic ester-containing surfactants are very stable in acidic environment but they undergo hydrolysis under neutral conditions [19,32]. On the basis of the experimental equilibrium surface tension isotherms, the critical micelle concentration (CMC) and minimum surface tension $\left(\gamma_{\min }\right)$ were determined to characterize the surface activity of the mixtures. For both surfactants in Hepes buffer solutions, the CMC values were $0.6 \mathrm{mM}$ (DMPM-11) and $0.75 \mathrm{mM}$ (DMM-11). Nevertheless, regardless of the impurities in the surfactants samples, the CMCs obtained for the DMM-11 (5.99 mM) and DMPM-11 (4 mM) dissolved in water were consistent with previously reported results (data not shown) [33]. In our opinion, the increased ionic strength can effectively reduce the electrostatic repulsion between the hydrophilic head groups. The surface tension curves (Fig. 1) corresponding to the surfactant-BSA complexes exhibit one break point. The concentration at the break point corresponds to the CMC of DMM-11 and DMPM-11 with BSA. The CMC values for the cationic surfactants DMM-11 and DMPM11 were higher in the presence of BSA, which can be explained considering the amount of surfactant that binds to the protein and the structural differences in the surfactant hydrophilic groups. Compared with the surfactant solutions without BSA, the CMCs of DMM-11 and DMPM-11 increased from $0.75 \mathrm{mM}$ to $0.87 \mathrm{mM}$ and from $0.6 \mathrm{mM}$ to $0.75 \mathrm{mM}$, respectively. The surfactant-BSA systems indicate that BSA molecules at the interface are gradually displaced by DMM-11 and DMPM-11 molecules due to competition at the adsorption layer. The number of surfactant molecules bound to the protein was estimated at the CMC. The results presented in Fig. 1 also indicate that although both CMCs for surfactant-BSA complexes were higher than those corresponding to the surfactants in Hepes buffer, there are more surfactant molecules of DMPM-11 interacting with BSA when compared to the DMM- 11 system once the saturation is reached (Table S2). In our opinion, the complex could have been formed between the negatively charged moieties of BSA and the positively charged head groups of DMM-11 and DMPM-11. Consequently, surfactants attached to the different functional sites, through electrostatic interactions, stay farther apart at the moment at which BSA unfolds.

\subsection{Fluorescence measurements}

Subsequently, fluorescence spectroscopy was used to evaluate the integrity of the protein structure. BSA contains two tryptophan residues (Trp135 and Trp214), the one located at position 135 is buried in a hydrophobic pocket, and it has been proposed to be located near the surface of the albumin molecule in the second helix of the first domain [34]. As BSA contains tryptophan (Trp) and tyrosine (Tyr) residues, its fluorescence emission by excitation at $280 \mathrm{~nm}$ should be dominated by the fluorescence of Trp residues due to the intramolecular energy transfer from Tyr to Trp residues [35]. To explore the surfactant-induced fluorescence change of BSA, measurements were performed at the excitation wavelength of $280 \mathrm{~nm}$. As shown in Fig. 2, the addition of DMM-11 and DMPM-11 significantly decreased the fluorescence intensity of BSA and both cationic surfactants, exhibited a similar behavior. As the surfactant concentration increased, the 

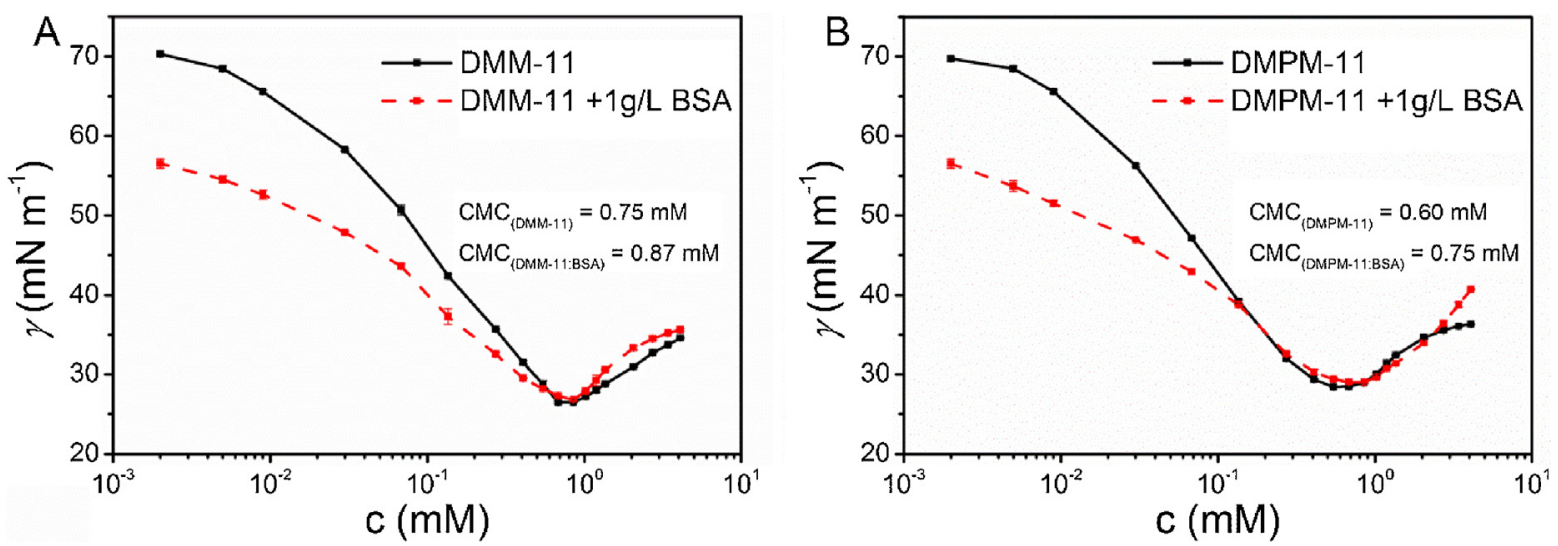

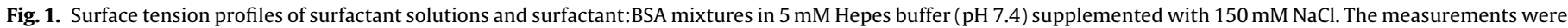
performed at $25^{\circ} \mathrm{C}$. (A) DMM-11; (B) DMPM-11. The values represent the mean of triplicates $\pm S D$.
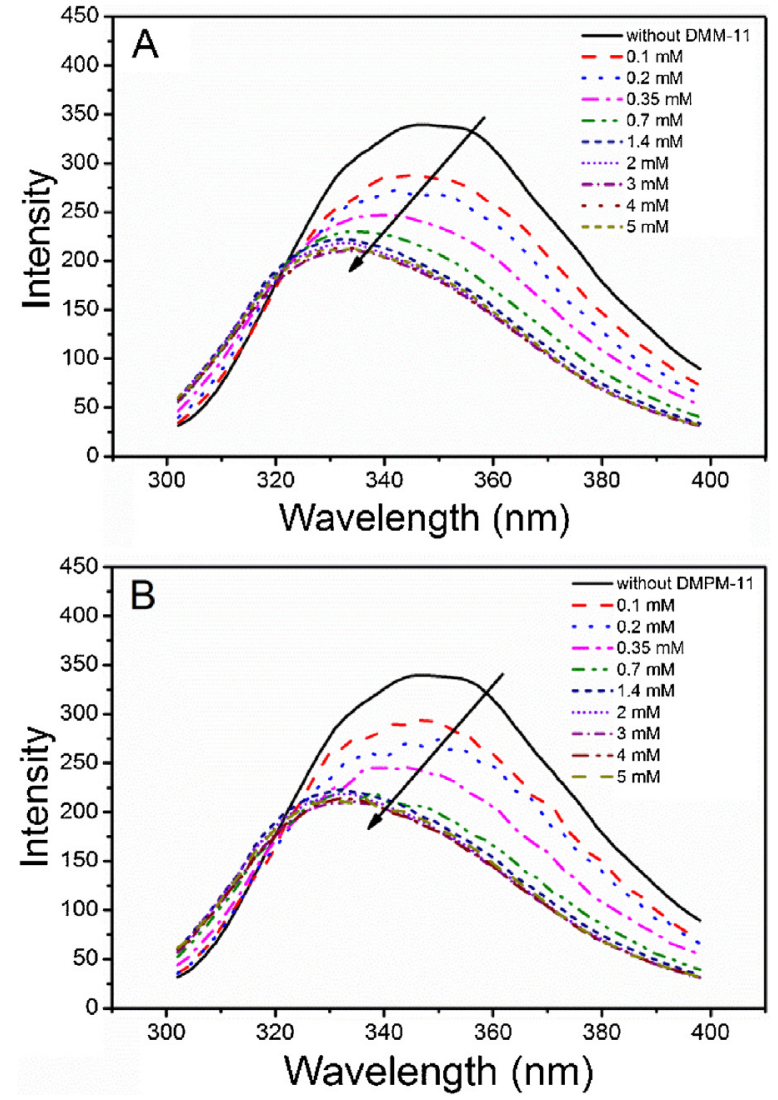

Fig. 2. Fluorescence emission spectra obtained for the surfactant-BSA systems excited at $280 \mathrm{~nm}\left(\mathrm{pH}=7.40 ; 25^{\circ} \mathrm{C}\right)$. BSA $(15 \mu \mathrm{M})$ in the presence of different concentrations of DMM-11 (A) and DMPM-11 (B).

fluorescence intensity decreased and a blue shift from 348 to $335 \mathrm{~nm}$ was observed in the maximal intensity. At low concentrations (below the CMC), the addition of DMM-11 and DMPM-11 to the BSA solutions caused a steep decrease in fluorescence emission, followed by a slower blue shift at higher concentrations of both surfactants (Fig. 2). Using the fluorescence quenching of Trp, Patel and co-workers [36] studied the interaction of the cationic gemini surfactant dodecyl betainate with lysozyme. The authors concluded that fluorescence intensity decreases significantly with the increase of the cationic gemini surfactant concentration. A similar trend has been reported in single-chain surfactant-BSA interactions, such as cationic DTAB-BSA [34] and TTAB-BSA [18].
Recent studies revealed that gemini surfactants quench the fluorescence intensity of gramicidin through a static mechanism [37]. Surfactant-induced fluorescence quenching of BSA is attributed to the formation of complexes between BSA and cationic surfactants. This binding perturbs the microenvironment around the Trp and Tyr residues and causes fluorescence quenching of the protein. The blue shift (decrease) of fluorescence band with increasing concentrations of DMM-11 and DMPM-11, suggests that the Trp residues of BSA are moved into a more hydrophobic environment.

Synchronous fluorescence spectroscopy is frequently used to characterize the interactions between surfactants and proteins, since it provides information about the molecular environment in the vicinity of the surfactant. Since the maximum emission wavelength of residues is related to its environmental polarity, the change of the protein conformation can be related to the emission wavelength change [38]. As shown in Fig. 3, both cationic surfactants increase the fluorescence intensity of $\operatorname{Tyr}(\Delta \lambda=20 \mathrm{~nm})$, and decrease the fluorescence intensity of $\operatorname{Trp}(\Delta \lambda=60 \mathrm{~nm})$. The fluorescence intensity of Trp residues was significantly higher than that of Tyr residues, indicating that the main contribution of the intrinsic fluorescence of BSA is due to the Trp residues, thus DMM11 and DMPM-11 mainly interact with Trp residues of BSA. These results are similar to those reported for the fluorescence intensity changes of BSA with quaternary ammonium cationic surfactants [39]. Furthermore, Wu and co-workers observed that the fluorescence intensity increases significantly with the increase of cationic gemini surfactants concentrations, when $\Delta \lambda$ was set at $20 \mathrm{~nm}$ [31].

\subsection{CD spectroscopy analysis}

The absorbance of peptide bonds is usually located in the far ultraviolet range; therefore, the absorption peak in the far ultraviolet range can reflect the occurrence of conformational changes (secondary structure) in the peptide chains of proteins. The effect of the cationic surfactants under study on the secondary structure of BSA was evaluated by CD spectroscopy. The CD spectra of studied systems were measured by monitoring the changes of the signal from 200 to $260 \mathrm{~nm}$. Fig. 4 shows the effect of DMM-11 and DMPM-11 on the CD spectrum of BSA. The CD spectra (Fig. 4) show mainly the shape of the $\alpha$-helical secondary structure and this can be connected with the negative ellipticity bands near $209 \mathrm{~nm}$. An increase in the concentration of both cationic surfactants results in an increment in the $\Delta \varepsilon$ values, both at 209 and $222 \mathrm{~nm}$. This suggests that the content of $\alpha$-helix is increased. To estimate the secondary structure composition, the spectra (with different molar extinction coefficients, $\Delta \varepsilon$ ) were analyzed by the K2D3 method [21]. Fitting the spectrum of native BSA at $25^{\circ} \mathrm{C}$ in Hepes buffer 

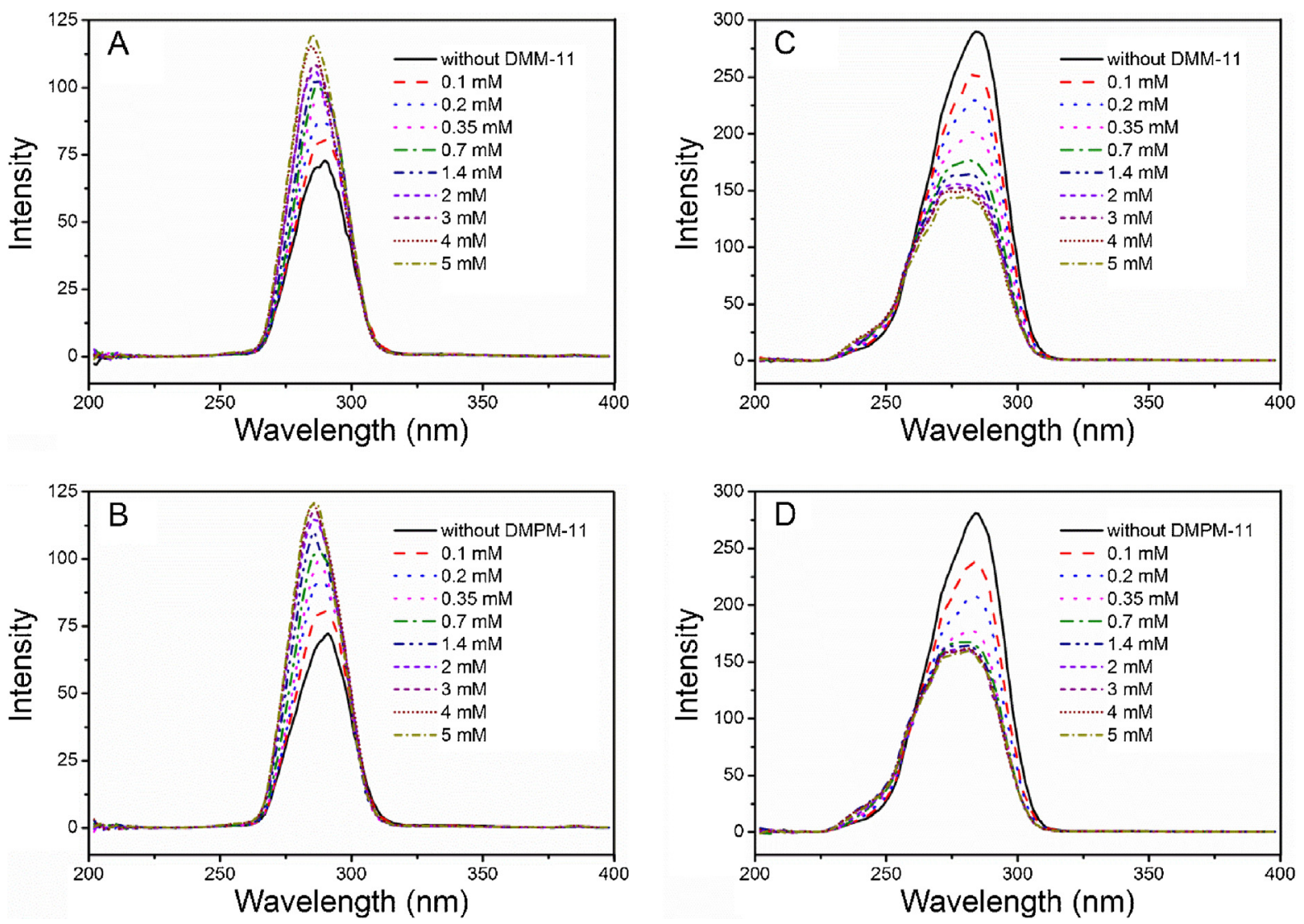

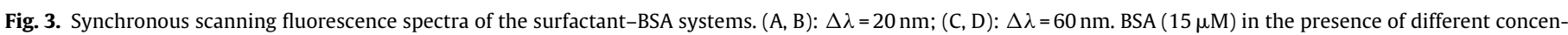
trations of DMM-11 (A, C) and DMPM-11 (B, D).

( $\mathrm{pH}=7.4)$ yielded a $68.59 \% \alpha$-helical structure. The addition of DMM-11 and DMPM-11 to BSA at the pre-micellar concentrations $(0.7 \mathrm{mM})$ caused a secondary structural change of the protein to $67.12 \% \alpha$-helical and $68.22 \% \alpha$-helical, respectively. According to the literature, the secondary structure of BSA may be stabilized by a cross-linking function at low concentrations of cationic surfactants [31,34]. Because DMM-11 and DMPM-11 exhibit an amphiphilic character, they may build bridges between particular negatively charged residues and particular nonpolar residues located on different loops of BSA. At higher concentrations of these surfactants, free micelles begin to form after the saturation binding of BSA with DMM-11 and DMPM-11, leading to BSA in an extended structure with exposed hydrophobic residues. The addition of sub-CMC concentrations $(2 \mathrm{mM})$ of DMM-11 and DMPM-11 to BSA caused a secondary structural change of the protein to $64.44 \% \alpha$-helical and $62.63 \% \alpha$-helical, respectively.

$\mathrm{CD}$ enables measuring the overall structural changes that BSA undergoes, unlike fluorescence, which reports the changes in and around the nearby environment of the surfactants [40]. Nevertheless, upon interaction with surfactants, the protein has a tendency to change its secondary structure, which has been clearly demonstrated (see Fig. 4). CD results are in very good agreement with the steady-state and time-resolved data (data not shown).

\subsection{ITC measurements}

Binding of DMM-11 and DMPM-11 to BSA was quantitatively studied by isothermal titration calorimetry (ITC), which provided data about the stoichiometry of binding: $n$ (number of surfactant molecules per one protein molecule), $K$ (binding constant), and $\Delta \mathrm{H}$ (binding enthalpy). The obtained enthalpy profiles together with the fitting parameters are presented in Fig. 5, Fig. 6 and Table S3. The top panel in Figs. 5 and 6 shows the raw baseline-smoothed ITC data plotted as heat flow versus time, whereas the bottom panel shows the integrated and concentration-normalized heat for each injection versus the surfactant/BSA molar ratio. The solid line in the lower panel represents the best fit of the data using the independent (one-site) or multiple sites (two-sites) models. The data obtained for BSA revealed a significant change of the enthalpy profile upon rising the surfactant concentration, which covers the pre-micellar concentration range $(<\mathrm{CMC})$ and the post-micellar concentration range $(>\mathrm{CMC})$. The strong influence of surfactant concentration on the process of association to the protein is a well-known fact, and it was also noticed while studying by ITC the influence of surfactants on BSA under concentration conditions of both pre-micellar [41-43] and post-micellar stages [43-46].

Fitting data for DMM-11 $\rightarrow$ BSA and DMPM-11 $\rightarrow$ BSA titrations showed that, at low concentrations of surfactant $(\leq 1.3 \mathrm{mM}$ for DMM-11 and $\leq 2 \mathrm{mM}$ for DMPM-11) (Figs. $5 \mathrm{~A}, 6 \mathrm{~A}$ ), one binding event occurs, which leads to the creation of a surfactant-BSA monomer. The best fit was obtained by one-site binding model $\left(\mathrm{n}_{1}\right.$ DMM-11,DMPM-11 $\approx 1$ ). Since in both cases those are entropy driven processes $(\Delta S>0)$ one could assume that the association is based on hydrophobic interactions [46]. At low surfactant concentration $(<\mathrm{CMC})$ the protein-surfactant interaction is specific in nature and only high affinity sites of the protein are occupied by surfactant molecules [42].

Upon the increase of the surfactant concentrations in the studied systems up to $5 \mathrm{mM}$, the enthalpy profiles (Figs. 5 B, 6 B) showed additional negative thermal effect, suggesting, apart from specific monomeric binding, the contribution of one more equilibrium. Since the surfactant concentration increases to post-micellar level, 

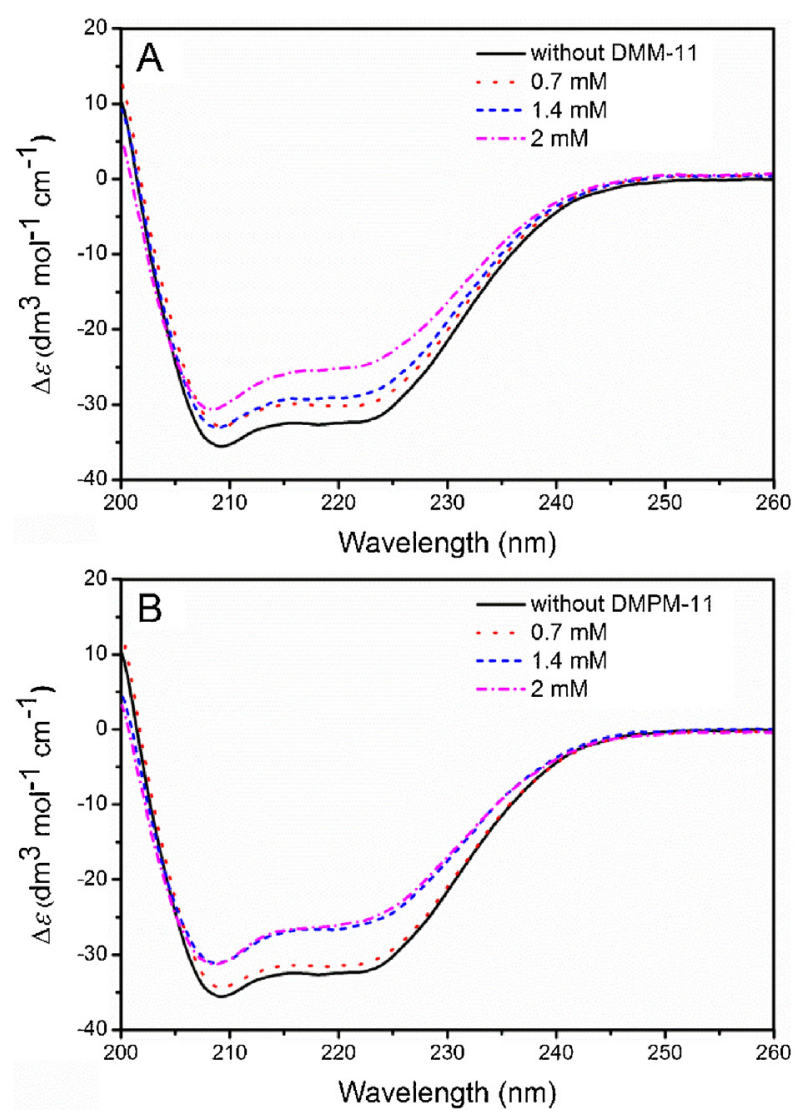

Fig. 4. Circular dichroism (CD) spectra of BSA $(1 \mathrm{~g} / \mathrm{L})$ alone and in the presence of different concentrations of DMM-11 (A) and DMPM-11 (B).

most likely it can be assigned to further binding of surfactants or to the conformational changes of the protein. For both systems, the 'two-sites' model was used to fit the titration points; the first site revealed again the monomeric stoichiometry, and the latter suggests the association of a higher number of surfactant molecules ( $\mathrm{n}_{2 \text { DMM-11 }} \approx 6.8, \mathrm{n}_{2}$ DMPM-11 $\approx 9.2$ ). The entropy of the process is positive. The binding constant $(K)$ of the second site is, for both surfactants, one order of magnitude lower than for the first binding site (Figs. 5 and 6), suggesting the 'non-specific' interactions of surfactant micelles with BSA (Table S3).

\subsection{Aggregation properties determined using DLS and DFT methods}

Our main goal was to understand the effect of the aggregation of DMM-11 and DMPM-11. The polydispersity indexes (PdI), hydrodynamic radii $\left(R_{\mathrm{H}}{ }^{\mathrm{DLS}}\right)$ and micelle volumes $\left(V_{\mathrm{mic}}{ }^{\mathrm{DLS}}\right)$ for DMM-11 and DMPM-11, along with their theoretically calculated counterparts $\left(R_{\mathrm{H}}{ }^{\mathrm{DFT}}, V_{\mathrm{mic}}{ }^{\mathrm{DFT}}\right)$ and aggregation numbers $\left(N_{\mathrm{agg}}{ }^{\mathrm{DFT}}\right)$, are shown in Table 1 . The PdI values were found to be close to 0.02 , indicating the monodisperse character of both cationic surfactants in water and Hepes buffer. This means that the surfactants under study form similar micelles, which is especially desirable for pharmaceutical applications.

As it can be seen from Table 1, a good agreement was observed between the predicted $\left(R_{\mathrm{H}}{ }^{\mathrm{DFT}}, V_{\mathrm{mic}}{ }^{\mathrm{DFT}}\right)$ and the observed values $\left(R_{\mathrm{H}}{ }^{\mathrm{DLS}}, V_{\text {mic }}{ }^{\mathrm{DLS}}\right)$. The $R_{\mathrm{H}}{ }^{\text {DLS }}$ and $R_{\mathrm{H}}{ }^{\mathrm{DFT}}$ of DMM-11 and DMPM-11 were $\sim 2 \mathrm{~nm}$, both in water and Hepes buffer. The $N_{\text {agg }}{ }^{\text {DFT }}$ values of DMPM-11 micelles are smaller than those corresponding to DMM-11 micelles in water. However, in Hepes buffer, both types of micelles are constituted exactly by the same number of molecules (Table 1).
DLS is a powerful technique that yields important structural information about biological macromolecules in solution. In fact, monitoring the size of a protein in the presence of surfactants can be used to investigate their interactions. In order to confirm the variation of the morphology and the hydrodynamic diameter $\left(D_{\mathrm{H}}\right)$ of BSA due to the presence of both cationic surfactants under study, DLS measurements were performed using BSA solutions $(1 \mathrm{~g} / \mathrm{L})$ with different concentrations of DMM-11 and DMPM-11. Fig. S3 shows the variation of $D_{\mathrm{H}}$ of BSA as a function of DMM-11 and DMPM-11 concentrations. The $D_{\mathrm{H}}$ of BSA increased at low concentrations (below the CMC) of DMM-11 and DMPM-11, from $7.1 \mathrm{~nm}$ to 9.1 and $8.8 \mathrm{~nm}$, respectively. Above the CMC, the $D_{\mathrm{H}}$ of BSA decreased from $\sim 9 \mathrm{~nm}$ to 7.5 and $7.2 \mathrm{~nm}$ with increasing DMM-11 and DMPM-11 concentrations, respectively.

The difference in the $D_{\mathrm{H}}$ of different BSA-surfactant complexes can be attributed to the difference in the number of cationic surfactants binding to BSA. On this basis, it can be inferred that a surfactant-BSA complex is formed and aggregates as the surfactant concentration increases. This inference was confirmed by the studies of the fluorescence intensity of BSA (Fig. 2) and the CD analysis for surfactant-BSA complexes (Fig. 4). These results imply that the binding between cationic surfactants and BSA leads to a conformational change in BSA which contributes to the aggregation of the DMM-11 $\rightarrow$ BSA and DMPM-11 $\rightarrow$ BSA complexes.

\subsection{Lysosomotropism evaluation}

The possibility and rate of penetration through biological membranes and, consequently, the probability of achieving an appropriate concentration in the region of action, is referred to LogP (partition coefficient of the neutral species of a compound between octanol and water). This value determines the absorption and the permeability across cell membranes. A recent screening of lysosomotropism found that drugs with a $\log \mathrm{P}>2$ cause lysosomal accumulation [47]. The calculated LogP for DMM-11 and DMPM11 are 3.96 and 4.25 , respectively. Another important parameter indicating bioconcentration is LogBCF. It refers to the process of uptake and buildup of chemicals in living organisms. The calculated $\log B C F=-2.74707$ (for DMM-11) and $\operatorname{LogBCF}=-2.89961$ (for DMPM-11) suggests that cationic surfactants will not accumulate in living organisms.

On the other hand, DMM-11 and DMPM-11 can be used in many other areas of medical sciences. DMM-11 and DMPM-11 are characterized by many biological activities and exhibit properties such as Anti-Herpes Simplex virus activity, Anti-Adenovirus activity, AntiInfluenza Birds activity and Anti-Oxidant activity, among others (Fig. S4). These calculations were obtained using the on-line software developed by ChemoSophia company.

In this sense, DMM-11 and DMPM-11 appear as particularly interesting molecules because they not only share the ability of lysosomotropic agents in general but also, being amphiphilic molecules, spontaneously organize in aggregates that could serve as carrier systems [48]. In order to exploit the targeting and biological properties of DMM-11 and DMPM-11, a deep understanding of their physicochemical behavior was required. Therefore, it was necessary to carry out studies using the model BSA protein. These studies provided useful and complementary information on the physiological regulation of cationic surfactants.

\section{Conclusion}

In the current work several techniques were used to unravel the nature of binding of cationic surfactants with different head groups (DMM-11 and DMPM-11) to the circulatory protein BSA. It was found that both cationic surfactants can interact with BSA, and 
A

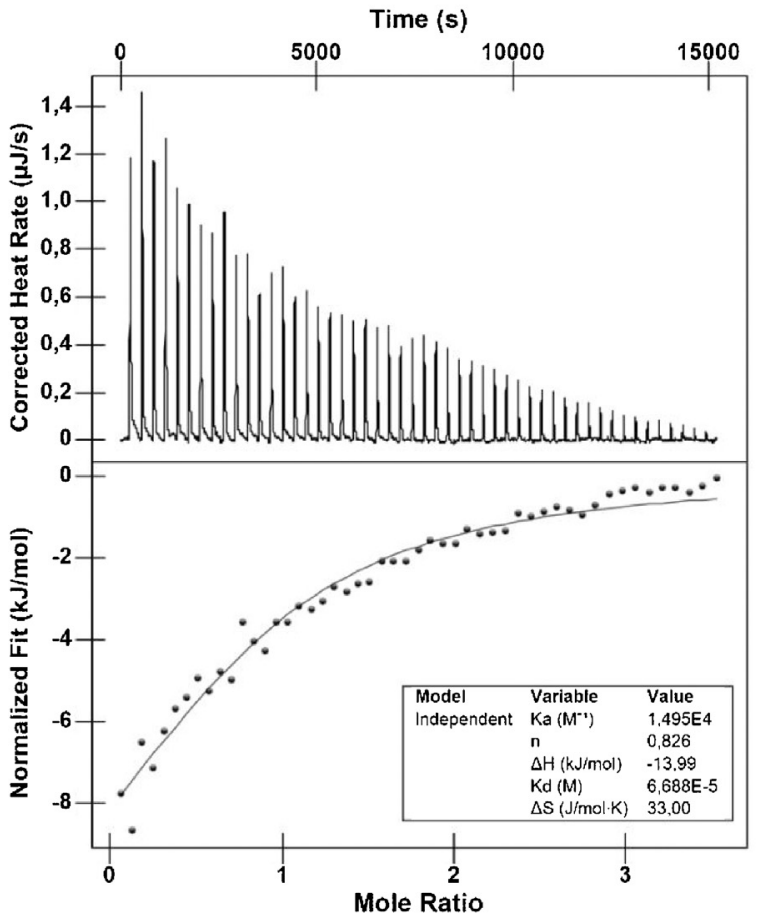

B

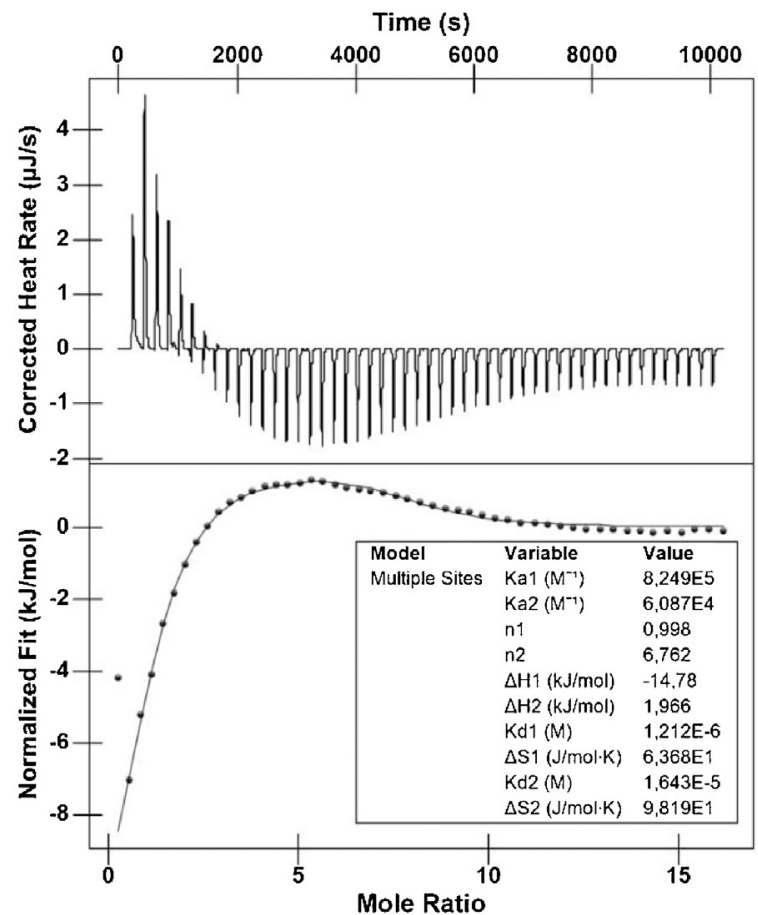

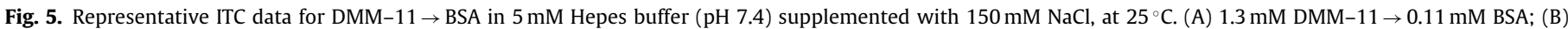
$5.0 \mathrm{mM}$ DMM-11 $\rightarrow 0.09 \mathrm{mM}$ BSA.

A

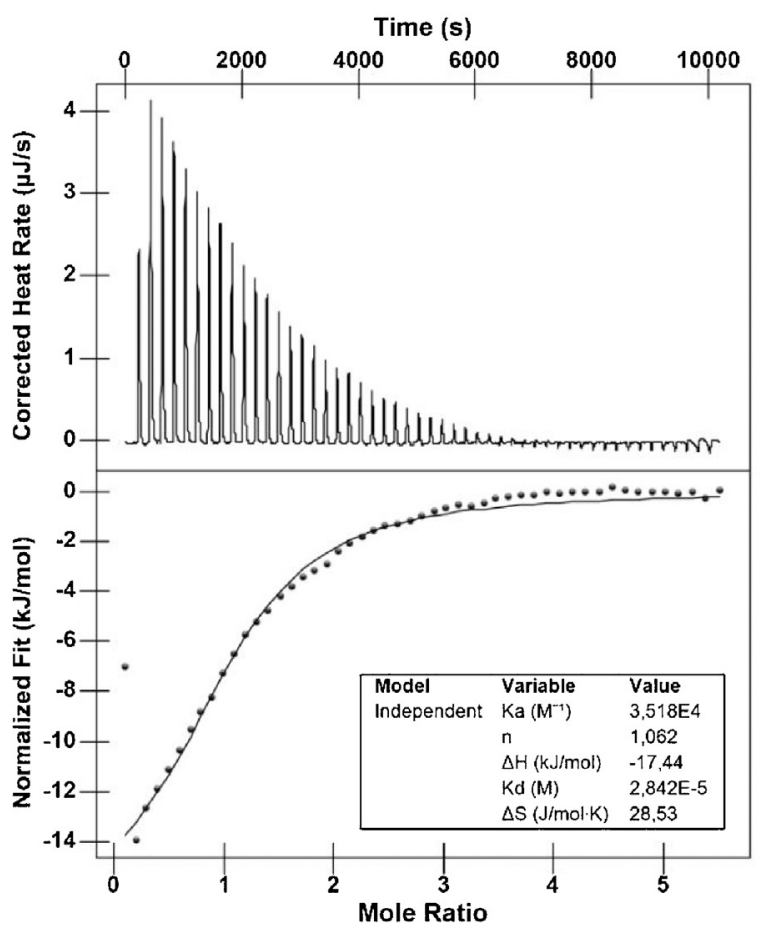

B

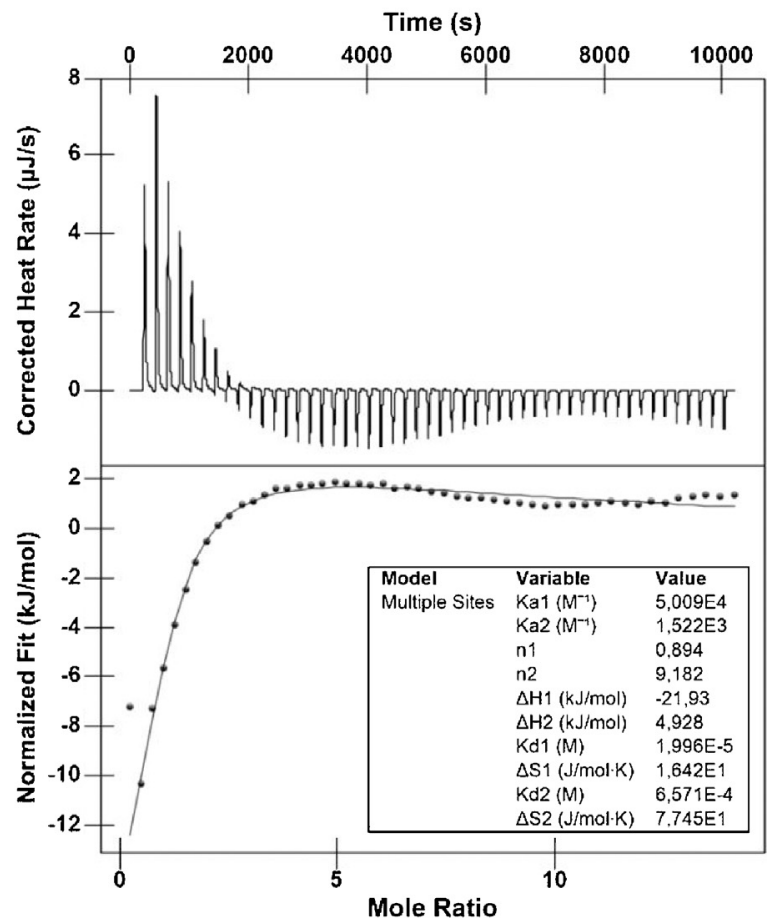

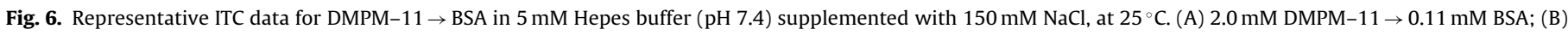
$5.1 \mathrm{mM}$ DMPM- $11 \rightarrow 0.11 \mathrm{mM}$ BSA.

the major binding mechanisms involve electrostatic and hydrophobic interactions. Synchronous fluorescence and CD spectra of BSA revealed that the conformation and microenvironment of the protein changes due to the binding of DMM-11 and DMPM-11. At low surfactant concentrations the interactions are estimated to be mainly electrostatic, but when the surfactant concentration increases, hydrophobic influence on the binding rises. The data 
Table 1

Selected properties of the micellar systems studied. Experimental results represent the mean of 9 replicates \pm SD.

\begin{tabular}{|c|c|c|c|}
\hline \multicolumn{4}{|l|}{ DLS } \\
\hline water & $\begin{array}{l}\text { PdI } \\
R_{\mathrm{H}}{ }^{(\mathrm{DLS})}[\mathrm{nm}] \\
V_{\mathrm{mic}}{ }^{(\mathrm{DLS})}\left[\mathrm{nm}^{3}\right]\end{array}$ & $\begin{array}{l}\text { DMM-11 } \\
0.034 \pm 0.001 \\
2.05 \pm 0.01 \\
36.09\end{array}$ & $\begin{array}{l}\text { DMPM-11 } \\
0.078 \pm 0.002 \\
2.01 \pm 0.01 \\
34.02\end{array}$ \\
\hline hepes & $\begin{array}{l}\text { PdI } \\
R_{\mathrm{H}}{ }^{(\mathrm{DLS})}[\mathrm{nm}] \\
V_{\mathrm{mic}}{ }^{(\mathrm{DLS})}\left[\mathrm{nm}^{3}\right]\end{array}$ & $\begin{array}{l}0.019 \pm 0.003 \\
2.07 \pm 0.02 \\
37.15\end{array}$ & $\begin{array}{l}0.017 \pm 0.001 \\
2.05 \pm 0.01 \\
36.08\end{array}$ \\
\hline $\begin{array}{l}\text { DFT } \\
\text { vaq }\end{array}$ & $\begin{array}{l}R_{\mathrm{H}}{ }^{(\mathrm{DFT})}[\mathrm{nm}] \\
V_{\text {mon }}{ }^{(\mathrm{DFT})}\left[\mathrm{nm}^{3}\right] \\
V_{\text {mic }}{ }^{(\mathrm{DFT})}\left[\mathrm{nm}^{3}\right] \\
N_{\text {agg }}{ }^{(\mathrm{DFT})}\end{array}$ & $\begin{array}{l}1.99 \\
0.48 \\
33.40 \\
69\end{array}$ & $\begin{array}{l}1.93 \\
0.43 \\
30.11 \\
70\end{array}$ \\
\hline water & $\begin{array}{l}R_{\mathrm{H}}{ }^{(\mathrm{DFT})}[\mathrm{nm}] \\
V_{\mathrm{mon}}{ }^{(\mathrm{DFT})}\left[\mathrm{nm}^{3}\right] \\
V_{\mathrm{mic}}{ }^{(\mathrm{DFT})}\left[\mathrm{nm}^{3}\right] \\
N_{\mathrm{agg}}{ }^{(\mathrm{DFT})}\end{array}$ & $\begin{array}{l}1.98 \\
0.42 \\
32.51 \\
77\end{array}$ & $\begin{array}{l}1.95 \\
0.43 \\
31.05 \\
72\end{array}$ \\
\hline$V_{\mathrm{mic}}^{(\mathrm{DLS})}=$ & $\begin{array}{l}R_{\mathrm{H}}{ }^{(\mathrm{DFT})}[\mathrm{nm}] \\
V_{\mathrm{mon}}{ }^{(\mathrm{DFT})}\left[\mathrm{nm}^{3}\right] \\
V_{\mathrm{mic}}{ }^{(\mathrm{DFT})}\left[\mathrm{nm}^{3}\right] \\
N_{\mathrm{agg}}{ }^{(\mathrm{DFT})}\end{array}$ & $\begin{array}{l}1.98 \\
0.47 \\
32.51 \\
69\end{array}$ & $\begin{array}{l}1.97 \\
0.46 \\
32.02 \\
69 \\
00 \text { की के }\end{array}$ \\
\hline
\end{tabular}

herein presented open new insights into the binding of lysosomotropic surfactants to BSA.

The micelles derived from the surfactants under study exhibit spherical shape and small size (with a low aggregation number). Moreover, the low polydispersity indexes determined experimentally by DLS for the micellar solutions of DMM-11 and DMPM-11 indicate that the formed micelles are remarkably homogeneous in size. Furthermore, the tested cationic surfactants are characterized by good bioavailability and low accumulation in living organisms. This study demonstrates that these compounds have potential for applications in chemical, biological and medical sciences. The lysosomotropic activity of soft analogues DMM-11 and DMPM-11, together with their compatibility with BSA, make them promising alternatives to long-chain QACs in a wide range of medical applications.

\section{Conflict of interest}

Authors disclose no conflict of interest.

\section{Acknowledgments}

Author Żaneta Czyżnikowska gratefully acknowledge the allotment of the CPU time in Wroclaw Center of Networking and Supercomputing (WCSS). All the ITC experiments were performed by Joanna Gałęzowska in the Laboratory of Elemental Analysis and Structural Research, Faculty of Pharmacy and the Division of Laboratory Diagnostics, Wroclaw Medical University, supported by the ERDF Project within the Innovation Economy Operational Programme POIG.02.01.00-14-122/09". Ligia R. Rodrigues and Eduardo J. Gudiña acknowledge the Portuguese Foundation for Science and Technology (FCT) for the financial support under the scope of the strategic funding of UID/BIO/04469/2013 unit and COMPETE 2020 (POCI-01-0145-FEDER-006684) and BioTecNorte operation (NORTE-01-0145-FEDER-000004) funded by the European Regional Development Fund under the scope of Norte2020 Programa Operacional Regional do Norte. The authors also thank the FCT for the financial support under the scope of the Project
RECI/BBB-EBI/0179/2012 (FCOMP-01-0124-FEDER-027462). This work was supported by Polish-Portugal Executive Program for years 2017-2018.

\section{Appendix A. Supplementary data}

Supplementary data associated with this article can be found, in the online version, at http://dx.doi.org/10.1016/j.colsurfb.2017.08. 046.

\section{References}

[1] T. Loftsson, T. Thorsteinsson, M. Masson, Hydrolysis kinetics and QSAR investigation of soft antimicrobial agents, J. Pharm. Pharmacol. 57 (2005) $721-727$.

[2] T. Ivankovic, J. Hrenovic, Surfactants in the environment, Arh. Hig. Rada Toksikol. 61 (2010) 95-110.

[3] A.S. Inacio, A. Nunes, C. Milho, L.J. Mota, M.J. Borrego, J.P. Gomes, W.L. Vaz, O.V. Vieira, In vitro activity of quaternary ammonium surfactants against streptococcal, chlamydial, and gonococcal infective agents, Antimicrob. Agents Chemother. 60 (2016) 3323-3332.

[4] J. Luczynski, Aminoethylesters of Fatty Acids as Lysosomotropic Substances, In: Ph.D Thesis, Wroclaw University of Technology, Wroclaw, 2000.

[5] A. Krasowska, L. Chmielewska, R. Adamski, J. Luczynski, S. Witek, K. Sigler, The sensitivity of yeast and yeast-like cells to new lysosomotropic agents, Cell. Mol. Biol. Lett. 9 (2004) 675-683.

[6] E. Oblak, T.M. Lachowicz, J. Luczynski, S. Witek, Lysosomotropic N,Ndimethyl alpha-aminoacid $\mathrm{N}$-alkyl esters and their quaternary ammonium salts as plasma membrane and mitochondrial ATPases inhibitors, Cell. Mol. Biol. Lett. 7 (2002) 1121-1129.

[7] T. Thorsteinsson, M. Masson, K.G. Kristinsson, M.A. Hjalmarsdottir, H. Hilmarsson, T. Loftsson, Soft antimicrobial agents: synthesis and activity of labile environmentally friendly long chain quaternary ammonium compounds, J. Med. Chem. 46 (2003) 4173-4181.

[8] O.F. Kuzu, M. Toprak, M.A. Noory, G.P. Robertson, Effect of lysosomotropic molecules on cellular homeostasis, Pharmacol. Res. 117 (2017) 177-184.

[9] D. Ajloo, N. Mahmoodabadi, M. Ghadamgahi, A.A. Saboury, Spectroscopy and computational studies on the interaction of octyl, dodecyl, and hexadecyl derivatives of anionic and cationic surfactants with adenosine deaminase, J. Biomol. Struct. Dyn. 34 (2016) 1495-1511.

[10] D. Otzen, Protein-surfactant interactions: a tale of many states, Biochim. Biophys. Acta 1814 (2011) 562-591.

[11] X. Vecino, J.M. Cruz, A.B. Moldes, L.R. Rodrigues, Biosurfactants in cosmetic formulations: trends and challenges, Crit. Rev. Biotechnol. (2017) 1-16.

[12] J.K. Maurya, M.U. Mir, N. Maurya, N. Dohare, A. Ali, R. Patel, A spectroscopic and molecular dynamic approach on the interaction between ionic liquid type 
gemini surfactant and human serum albumin, J. Biomol. Struct. Dyn. 34 (2016) 2130-2145.

[13] V.K. Vishvakarma, K. Kumari, R. Patel, V.S. Dixit, P. Singh, G.K. Mehrotra, R. Chandra, A.K. Chakrawarty, Theoretical model to investigate the alkyl chain and anion dependent interactions of gemini surfactant with bovine serum albumin, Spectrochim. Acta. A. Mol. Biomol. Spectrosc. 143 (2015) 319-323.

[14] M.U.H. Mir, J.K. Maurya, S. Ali, S. Ubaid-Ullah, A.B. Khan, R. Patel, Molecular interaction of cationic gemini surfactant with bovine serum albumin: a spectroscopic and molecular docking study, Process Biochem. 49 (2014) 623-630.

[15] S. Sinha, D. Tikariha, J. Lakra, T. Yadav, S. Kumari, S.K. Saha, K.K. Ghosh, Interaction of bovine serum albumin with cationic monomeric and dimeric surfactants: a comparative study, J. Mol. Liq. 218 (2016) 421-428.

[16] M. Kongot, N. Maurya, N. Dohare, M. u. d. Parray, J.K. Maurya, A.R. Kumar Patel, Enthalpy-driven interaction between dihydropyrimidine compound and bovine serum albumin: a spectroscopic and computational approach, J. Biomol. Struct. Dyn. (2017) 1-10.

[17] Y. Liu, Z. Cao, J. Wang, W. Zong, R. Liu, The interaction mechanism between anionic or cationic surfactant with HSA by using spectroscopy, calorimetry and molecular docking methods, J. Mol. Liq. 224 (2016) 1008-1015.

[18] P.K. Misra, U. Dash, S. Maharana, Investigation of bovine serum albumin-surfactant aggregation and its physicochemical characteristics, Colloids Surf. Physicochem. Eng. Aspects 483 (2015) 36-44.

[19] G. Para, J. Luczynski, J. Palus, E. Jarek, K.A. Wilk, P. Warszynski, Hydrolysis driven surface activity of esterquat surfactants, J. Colloid Interface Sci. 465 (2016) 174-182.

[20] C. Huh, S.G. Mason, A rigorous theory of ring tensiometry, Colloid. Polym. Sci 253 (1975) 566-580

[21] C. Louis-Jeune, M.A. Andrade-Navarro, C. Perez-Iratxeta, Prediction of protein secondary structure from circular dichroism using theoretically derived spectra, Proteins 80 (2012) 374-381.

[22] A.D. Becke, Density-functional exchange-energy approximation with correct asymptotic behavior, Phys. Rev. A 38 (1988) 3098.

[23] C. Lee, W. Yang, R.G. Parr, Development of the Colle-Salvetti correlation-energy formula into a functional of the electron density, Phys. Rev. B 37 (1988) 785.

[24] T. Yanai, D.P. Tew, N.C. Handy, A new hybrid exchange-correlation functional using the Coulomb-attenuating method (CAM-B3LYP), Chem. Phys. Lett. 393 (2004) 51-57.

[25] V. Barone, M. Cossi, Quantum calculation of molecular energies and energy gradients in solution by a conductor solvent model, J. Phys. Chem. A 102 (1998) 1995-2001.

[26] R. Improta, V. Barone, G. Scalmani, M.J. Frisch, A state-specific polarizable continuum model time dependent density functional theory method for excited state calculations in solution, J. Chem. Phys. 125 (2006) 054103.

[27] M. Frisch, G.W. Trucks, H.B. Schlegel, G.E. Scuseria, M.A. Robb, J.R. Cheeseman, G. Scalmani, V. Barone, B. Mennucci, G.A. Petersson, Gaussian 09, 270, Gaussian Inc., Wallingford, CT, 2009, pp. 271 (revision A. 02).

[28] V.A. Potemkin, M.A. Grishina, A new paradigm for pattern recognition of drugs, J. Comput. Aided Mol. Des. 22 (2008) 489-505.

[29] V. Potemkin, M. Grishina, Principles for 3D/4D QSAR classification of drugs, Drug Discov. Today 13 (2008) 952-959.

[30] V.A. Potemkin, A.A. Pogrebnoy, M.A. Grishina, Technique for energy decomposition in the study of receptor-ligandcomplexes, J. Chem. Inf. Model. 49 (2009) 1389-1406.

[31] D. Wu, G. Xu, Y. Sun, H. Zhang, H. Mao, Y. Feng, Interaction between proteins and cationic gemini surfactant, Biomacromolecules 8 (2007) 708-712.
[32] A.R. Tehrani-Bagha, H. Oskarsson, C.G. van Ginkel, K. Holmberg, Cationic ester-containing gemini surfactants: chemical hydrolysis and biodegradation, J. Colloid Interface Sci. 312 (2007) 444-452.

[33] K. Dopierala, K. Prochaska, Dynamics of adsorption in micellar and non micellar solutions of derivatives of lysosomotropic substances, Adv. Colloid Interface Sci. 156 (2010) 62-69.

[34] S.S. Madaeni, E. Rostami, Spectroscopic investigation of the interaction of BSA with cationic surfactants, Chem. Eng. Technol. 31 (2008) 1265-1271.

[35] A. Sułkowska, Interaction of drugs with bovine and human serum albumin, J. Mol. Struct. 614 (2002) 227-232.

[36] R. Patel, J.K. Maurya, M.U.H. Mir, M. Kumari, N. Maurya, An insight into the binding between ester-functionalized cationic Gemini surfactant and lysozyme, J. Lumin. 154 (2014) 298-304.

[37] R. Patel, M. ud din Parray, A. Singh, P. Venkatesu, S.H.B. Singh Bohidar, Effect of 1, 4-bis (3-dodecylimidazolium-1-yl) butane bromide on channel form of gramicidin vesicles, Colloids Surf. Physicochem. Eng. Aspects 508 (2016) $150-158$.

[38] Y.Z. Zhang, B. Zhou, Y.X. Liu, C.X. Zhou, X.L. Ding, Y. Liu, Fluorescence study on the interaction of bovine serum albumin with p-aminoazobenzene, J. Fluoresc. 18 (2008) 109-118.

[39] H.-J. Xie, C.-C. Liu, Q. Sun, Q. Gu, Q.-F. Lei, W.-J. Fang, The interactions between quaternary ammonium cationic surfactants and bovine serum albumin, Acta Phys. Chim. Sin 32 (2016) 2951-2960.

[40] N.K. Das, L. Pawar, N. Kumar, S. Mukherjee, Quenching interaction of BSA with DTAB is dynamic in nature: a spectroscopic insight, Chem. Phys. Lett. 635 (2015) 50-55.

[41] E.L. Gelamo, C.H. Silva, H. Imasato, M. Tabak, Interaction of bovine (BSA) and human (HSA) serum albumins with ionic surfactants: spectroscopy and modelling, Biochim. Biophys. Acta 1594 (2002) 84-99.

[42] S. Ghosh, J. Dey, Interaction of bovine serum albumin with N-acyl amino acid based anionic surfactants: effect of head-group hydrophobicity, J. Colloid Interface Sci. 458 (2015) 284-292.

[43] S.K. Singh, N. Kishore, Thermodynamic insights into the binding of Triton $\mathrm{X}-100$ to globular proteins: a calorimetric and spectroscopic investigation, J. Phys. Chem. B 110 (2006) 9728-9737.

[44] M. Liu, D. -z. Sun, B. Li, L.-Y. Zhu, Studies on the binding of Bis-quaternary ammonium surfactants to bovine serum albumins by microcalorimetry and circular dichroism, J. Dispers. Sci. Technol. 30 (2009) 459-465.

[45] A.D. Nielsen, K. Borch, P. Westh, Thermochemistry of the specific binding of C12 surfactants to bovine serum albumin, Biochim. Biophys. Acta 1479 (2000) 321-331.

[46] A. Zaragoza, J.A. Teruel, F.J. Aranda, A. Marques, M.J. Espuny, A. Manresa, A. Ortiz, Interaction of a Rhodococcus sp. trehalose lipid biosurfactant with model proteins: thermodynamic and structural changes, Langmuir 28 (2012) 1381-1390.

[47] S. Nadanaciva, S. Lu, D.F. Gebhard, B.A. Jessen, W.D. Pennie, Y. Will, A high content screening assay for identifying lysosomotropic compounds, Toxicol. In Vitro 25 (2011) 715-723.

[48] L.R. Rodrigues, Microbial surfactants: fundamentals and applicability in the formulation of nano-sized drug delivery vectors, J. Colloid Interface Sci. 449 (2015) 304-316. 